\title{
Biotransformation of major ginsenoside Rb1 to pharmacologically active ginsenoside Rg3 through fermentation byWeissella hellenica DC06 in newly developed medium
}

\author{
Md. A. Huq ${ }^{1}$, S. Akter ${ }^{2}$, Yeon-Ju Kim², Mohamed El-Agamy Farh ${ }^{1}$ and Deok-Chun Yang ${ }^{1 *}$ \\ ${ }^{I}$ Graduate School of Biotechnology and Ginseng Bank, College of Life Science, Kyung Hee University, Yongin, \\ 446-701, Republic of Korea \\ ${ }^{2}$ Department of Oriental Medicinal Material and Processing, College of Life Science, Kyung Hee University, \\ Yongin, 446-701, Republic of Korea
}

\begin{abstract}
The study was conducted to develop an edible and low cost growth medium for cultivation of Weissella hellenica DC06, a lactic acid bacteria (LAB) and to study whether, the medium is suitable for bioconversion of major ginsenoside Rb1 into ginsenoside Rg3 through fermentation by $W$. hellenica DC06. Fourteen different media compositions were investigated to cultivate $W$. hellenica DC06. Among these, W. hellenica DC06 exhibited the highest growth in media containing $20 \mathrm{~g} / 1$ radish, $20 \mathrm{~g} / 1$ glucose, and $10 \mathrm{~g} / 1$ yeast extract (Medium 3). The optical density of $W$. hellenica DC06 cultivated in medium 3 reached $1.8(1.066$ x $1010 \mathrm{CFU} / \mathrm{ml})$ after $24 \mathrm{~h}$ of incubation. Importantly, the optimized medium was approximately four times cheaper compared to MRS medium. In addition to being economical, the new medium was also edible. Also $W$. hellenica DC06 showed strong fermentation ability in newly developed medium regarding on major ginsenoside $\mathrm{Rb} 1$ biotransformation. Ginsenoside Rb1 was converted into pharmacologically active ginsenoside Rg3 in new medium. In contrast, $W$. hellenica DC06 showed weak fermentation ability in MRS medium where ginsenoside Rb1 was converted intoginsenoside Rd. The transformation products were analyzed by TLC, and HPLC. Within seven days of fermentation, almost all ginsenoside Rb1 was decomposed and converted into Rg3 in optimized medium. W. hellenica DC06 hydrolyzed two glucose moieties attached to the C-20 position of the ginsenoside Rb1aglyconeand synthesized $\operatorname{Rg} 3$ in newly developed medium.
\end{abstract}

Keywords:Economical medium; Weissellahellenica; Ginsenoside; Biotransformation.

\section{Introduction}

Ginsenoside, a major component of ginseng, has been reported to exhibit various biological effects such as anticancer, anti-obesity,tumor-suppressing, hepatoprotective, neuroprotective effects (Huq et al., 2015a; Lee et al., 2005; Shinkai et al., 1996; Tian et al., 2005). Minor ginsenosides are more active than intact major ginsenosides (Huq et al., 2015a). Therefore, many researchers have attempted to convert major ginsenosides to more active minor ginsenosides (Huq et al., 2014; Huq et al., 2015a,b,c). Researchers also have attempted to use lactic acid bacteria (LAB) for transformation of major ginsenosides into pharmacologically active minor ginsenosides because LAB has many positive effects on human health (Huq et al., 2014; Huq et al., 2015c). As
LAB cells are fastidious with respect to nutrient requirements, rich medium is required for good growth (Liew et al., 2005). The most common media for growth of LAB is Man Rogosa Sharpe medium (MRS) (de Man et al., 1960; Rogosa et al., 1961), which contains $25 \mathrm{~g} / \mathrm{l}$ of a complex nitrogen source consisting of peptone (protein hydrolysate) and extracts of both meat and yeast. However, the cost of MRS media is prohibitive for use in commercial applications (Zhang and Greasham, 1999). Another important disadvantage of MRS medium is the presence of constituents of bovine origin, which should be avoided in food production because of risks related to the occurrence of bovine spongiform encephalopathy (Wells et al., 1987).

*Corresponding author e-mail: deokchunyang@yahoo.co.kr 
Radishes (Raphanussativus L.) can be used as an inexpensive source of essential microbial nutrients for a variety of purposes. It is a short duration crop and has very high growth rates. Radishes contain mostly protein, sugar, and vitamins (vitamin $\mathrm{C}$, thiamine, riboflavin, niacin, pantothenic acid, vitamin B6, folate), as well as other important nutrients such as sodium, potassium, calcium, magnesium, phosphorus, iron, and zinc (USDA National Nutrient data base).

Weissella hellenica is a lactic acid bacterial species that is usually found in flounder intestine (Cai et al., 1998), kimchi (Kim et al., 2008), meat and meat products, and pickles, as well as some other sources. $W$. hellenica exhibits high antimicrobial activity by producing a bacteriocin called weissellicin that acts against pathogenic bacteria (Masuda et $a l ., 2012)$. In this study we isolated $W$. hellenica DC06 from a well-known Korean fermented food kimchi that is generally produce from radish and cabbage and developed an economical medium for cultivation of $W$. hellenica by using cheap natural sources. We also checked the major ginsenoside $\mathrm{Rb} 1$ fermentation ability of $W$. hellenica that was grown in new medium.

\section{Materials and methods}

The $W$. hellenica DC06 strain was isolated from kimchi.This strain has been deposited to the Korean Collection for Type Cultures (KCTC). Deposition number, KCTC 21039. Radish and cabbage were purchased from a local market. Glucose, sucrose, yeast extract, and MRS broth were purchased from Difco (Miller, Becton Dickinson and Co., MD, USA).Ginsenoside Rb1 and all standard ginsenosides were obtained from the Ginseng Genetic Resource Bank (Kyung Hee University, Yongin, Korea).

Preparation of media containing radish and cabbage powder

Radish and cabbage were cut into small pieces and dried in a $50^{\circ} \mathrm{C}$ oven for $24 \mathrm{~h}$. After drying, radish and cabbage powders were prepared using a blender, mixed with distilled $\mathrm{H}_{2} \mathrm{O}$, and boiled at $100^{\circ} \mathrm{C}$ for $15 \mathrm{~min}$. All of the nutrients from the radish and cabbage were extracted as a result of the boiling and heating procedures. The dissolved solutions were subjected to filtration after boiling to remove cell debris, which would have inhibited bacteria growth due to its high concentration. Finally, sugars and yeast extract were added to the filtered media and autoclavedat $121^{\circ} \mathrm{C}$ for 15 $\min$.

\section{Inoculum preparation}

For seed culture, $W$. hellenica DC06 was grown on MRS agar plates at $37^{\circ} \mathrm{C}$ for two days. Single colonies were selected from petri dishes and inoculated into MRS broth $(\mathrm{pH} 6.5)$ followed by incubation at $37^{\circ} \mathrm{C}$ with shaking $(160 \mathrm{rpm})$ overnight.

\section{Culture conditions}

Fourteen different media compositions were designed that containing varying amounts of radish, cabbage, sugars, and yeast extract (Table I) for cultivation of $W$. hellenica DC06 as described below. Cultivation was carried out in $100 \mathrm{ml}$ flasks with $50 \mathrm{ml}$ media for $72 \mathrm{~h}$. One $\mathrm{ml}$ of seed culture was inoculated in each medium, and the temperature was maintained at $37^{\circ} \mathrm{C}$ with shaking at $160 \mathrm{rpm}$. pH was maintained at 6.5 by using $\mathrm{NaOH}$. During incubation, growth was monitored by the measurement of OD (optical density) at $600 \mathrm{~nm}$ at $24 \mathrm{~h}$ intervals. In addition, growth in optimal medium was compared with that of MRS medium.

\section{Media optimization}

For optimization of media composition used different concentration of radish $(5 \mathrm{~g} / \mathrm{l}, 10 \mathrm{~g} / \mathrm{l}, 20 \mathrm{~g} / \mathrm{l}, 30 \mathrm{~g} / \mathrm{l})$, cabbage (5 $\mathrm{g} / 1,10 \mathrm{~g} / 1,20 \mathrm{~g} / \mathrm{l})$, glucose $(10 \mathrm{~g} / 1,20 \mathrm{~g} / \mathrm{l})$ and yeast extract $(5 \mathrm{~g} / 1,10 \mathrm{~g} / \mathrm{l})$.

Fermentation of major ginsenoside Rb1 by W. hellenica DC06 in newly developed medium

The fermentation procedure was carried out in a $50 \mathrm{ml}$ screw-cap tube. Single colonies of $W$. hellenica DC06 were selected from petri dishes and inoculated into $10 \mathrm{ml}$ of newly developed medium and then $2 \mathrm{mg}$ ginsenoside Rb1 was inserted into the medium. The mixture was then incubated at $37^{\circ} \mathrm{C}$ with shaking (160 rpm). Samples were withdrawn at regular intervals (every $24 \mathrm{~h}$ ), and water-saturated n-butanol was added to each sample to stop the reaction. Subsequently, the butanol fraction was allowed to evaporate completely, and the methanol extract was analyzed by TLC and HPLC. 
Table I. Growth rates of Weissella hellenica DC06 in different media compositions with the mean \pm standard deviation (SD) of replicates g/l.

\begin{tabular}{|c|c|c|c|c|c|c|c|c|}
\hline \multirow[t]{2}{*}{ Medium } & \multirow{2}{*}{$\begin{array}{l}\text { Radish } \\
(\mathrm{g} / 1)\end{array}$} & \multirow{2}{*}{$\begin{array}{c}\text { Cabbage } \\
(\mathrm{g} / 1)\end{array}$} & \multirow{2}{*}{$\begin{array}{l}\text { Glucose } \\
(\mathrm{g} / 1)\end{array}$} & \multirow{2}{*}{$\begin{array}{c}\text { Sucrose } \\
(\mathrm{g} / 1)\end{array}$} & \multirow{2}{*}{$\begin{array}{c}\text { Yeast } \\
\text { extract }(\mathrm{g} / 1)\end{array}$} & \multicolumn{3}{|c|}{ Optical density (OD) at 600nm } \\
\hline & & & & & & $24(\mathrm{~h})$ & $48(\mathrm{~h})$ & 72(h) \\
\hline 1 & 20.00 & 0.00 & 0.00 & 0.00 & 0.00 & $0.995 \pm 0.027$ & $1.097 \pm 0.134$ & $1.017 \pm 0.179$ \\
\hline 2 & 20.00 & 0.00 & 10.00 & 0.00 & 10.00 & $1.643 \pm 0.042$ & $1.756 \pm 0.063$ & $1.470 \pm 0.092$ \\
\hline 3 & 20.00 & 0.00 & 20.00 & 0.00 & 10.00 & $1.805 \pm 0.070$ & $1.950 \pm 0.098$ & $1.967 \pm 0.133$ \\
\hline 4 & 20.00 & 0.00 & 0.00 & 20.00 & 10.00 & $1.608 \pm 0.090$ & $1.676 \pm 0.069$ & $1.462 \pm 0.057$ \\
\hline 5 & 20.00 & 0.00 & 20.00 & 0.00 & 5.00 & $1.515 \pm 0.119$ & $1.666 \pm 0.050$ & $1.314 \pm 0.183$ \\
\hline 6 & 30.00 & 0.00 & 20.00 & 0.00 & 10.00 & $1.753 \pm 0.089$ & $1.886 \pm 0.083$ & $1.862 \pm 0.035$ \\
\hline 7 & 10.00 & 0.00 & 20.00 & 0.00 & 10.00 & $1.641 \pm 0.124$ & $1.783 \pm 0.067$ & $1.772 \pm 0.093$ \\
\hline 8 & 5.00 & 0.00 & 20.00 & 0.00 & 10.00 & $1.047 \pm 0.212$ & $0.957 \pm 0.096$ & $0.892 \pm 0.063$ \\
\hline 9 & 0.00 & 5.00 & 20.00 & 0.00 & 10.00 & $1.496 \pm 0.023$ & $1.497 \pm 0.011$ & $1.453 \pm 0.006$ \\
\hline 10 & 0.00 & 10.00 & 20.00 & 0.00 & 10.00 & $1.505 \pm 0.016$ & $1.719 \pm 0.134$ & $1.674 \pm 0.063$ \\
\hline 11 & 0.00 & 20.00 & 20.00 & 0.00 & 10.00 & $1.365 \pm 0.093$ & $1.511 \pm 0.111$ & $1.620 \pm 0.05 .7$ \\
\hline 12 & 5.00 & 5.00 & 20.00 & 0.00 & 10.00 & $1.594 \pm 0.074$ & $1.599 \pm 0.082$ & $1.572 \pm 0.064$ \\
\hline 13 & 10.00 & 10.00 & 20.00 & 0.00 & 10.00 & $1.728 \pm 0.070$ & $1.736 \pm 0.060$ & $1.683 \pm 0.065$ \\
\hline 14 & 10.00 & 5.00 & 20.00 & 0.00 & 10.00 & $1.667 \pm 0.062$ & $1.683 \pm 0.069$ & $1.672 \pm 0.064$ \\
\hline MRS & & & & & & $2.28 \pm 0.093$ & $2459 \pm 0.033$ & $2.496 \pm 0.032$ \\
\hline
\end{tabular}

\section{TLC analysis of ginsenosides}

TLC analysis was carried out using silica gel plates (60F254; Merck, Darmstadt, Germany) with a solvent system of $\mathrm{CHCl}_{3}: \mathrm{CH}_{3} \mathrm{OH}: \mathrm{H}_{2} \mathrm{O}(65: 35: 10 \mathrm{v} / \mathrm{v} / \mathrm{v})$ as the developer. The spots on the TLC plates were sprayed with $10 \%(\mathrm{v} / \mathrm{v}) \mathrm{H}_{2} \mathrm{SO}_{4}$ and heated at $110^{\circ} \mathrm{C}$ for $10 \mathrm{~min}$ before detection.

\section{HPLC analysis for biotransformation of ginsenosides}

HPLC-grade acetonitrile and water were purchased from SK Chemicals (Ulsan, Korea). The reaction mixture was extracted with $n$-butanol saturated with $\mathrm{H}_{2} \mathrm{O}$, evaporated in vacuo, and the residue was dissolved in $\mathrm{CH}_{3} \mathrm{OH}$ and applied to the HPLC analysis. HPLC was performed using a C18 (50 × 4.6 $\mathrm{mm}$, Kinetex 2.6u C18 100A) column with acetonitrile (solvent $\mathrm{A}$ ) and distilled water (solvent B) as the mobile phases with $85 \%$ B for $5 \mathrm{~min}, 79 \%$ B for $20 \mathrm{~min}, 42 \%$ B for $55 \mathrm{~min}$, $10 \% \mathrm{~B}$ for $12 \mathrm{~min}$, and $85 \% \mathrm{~B}$ for $18 \mathrm{~min}$, at a flow rate of $1 \mathrm{ml} / \mathrm{min}$. The sample was detected at UV $203 \mathrm{~nm}$.

\section{Statistical analysis}

All data are presented as mean \pm standard error (S.E.), and all experiments were independently performed three times.

\section{Results and discussion}

Determination of an economical medium

The growth activity of $W$. hellenica DC06 in 14 different media compositions varied significantly (Fig. 1). Among the 14 media types, $W$. hellenica DC06 exhibited the highest rate of growth in Medium 3, which contained $20 \mathrm{~g} / 1$ radish, $20 \mathrm{~g} / 1$ glucose, and $10 \mathrm{~g} / 1$ yeast extract. Specifically, after incubation for $24 \mathrm{~h} \mathrm{~W}$. hellenica DC06 had an optical density at 600 $\mathrm{nm}$ of 1.805 and $1.066 \times 10^{10} \mathrm{CFU} / \mathrm{ml}$. After 48 and $72 \mathrm{~h}$ optical density was near constant, reaching 1.950 and 1.967 , respectively. With respect to cabbage, we used $5 \mathrm{~g} / 1,10 \mathrm{~g} / 1$ and $20 \mathrm{~g} / 1$ cabbage with $20 \mathrm{~g} / 1$ glucose and $10 \mathrm{~g} / 1$ yeast extract; however, none of these composition resulted in better growth compared with Medium 3, which contained $20 \mathrm{~g} / 1$ radish. Here also used radish and cabbage together with glucose and yeast extract, but they did not exhibit as good of a growth rate as observed for Medium 3 (Fig. 1).

For optimization of radish containing media, four different concentrations of radish was tested with glucose $(20 \mathrm{~g} / \mathrm{l})$ and yeast extract $(10 \mathrm{~g} / \mathrm{l})$. Among these, $20 \mathrm{~g} /$ lradish produced maximal growth. Conversely, $30 \mathrm{~g} / 1$ radish resulted in a slightly lower growth rate (OD, 1.75) after $24 \mathrm{~h}$ of incubation. Likewise, growth of $W$. hellenica DC06 in media con- 


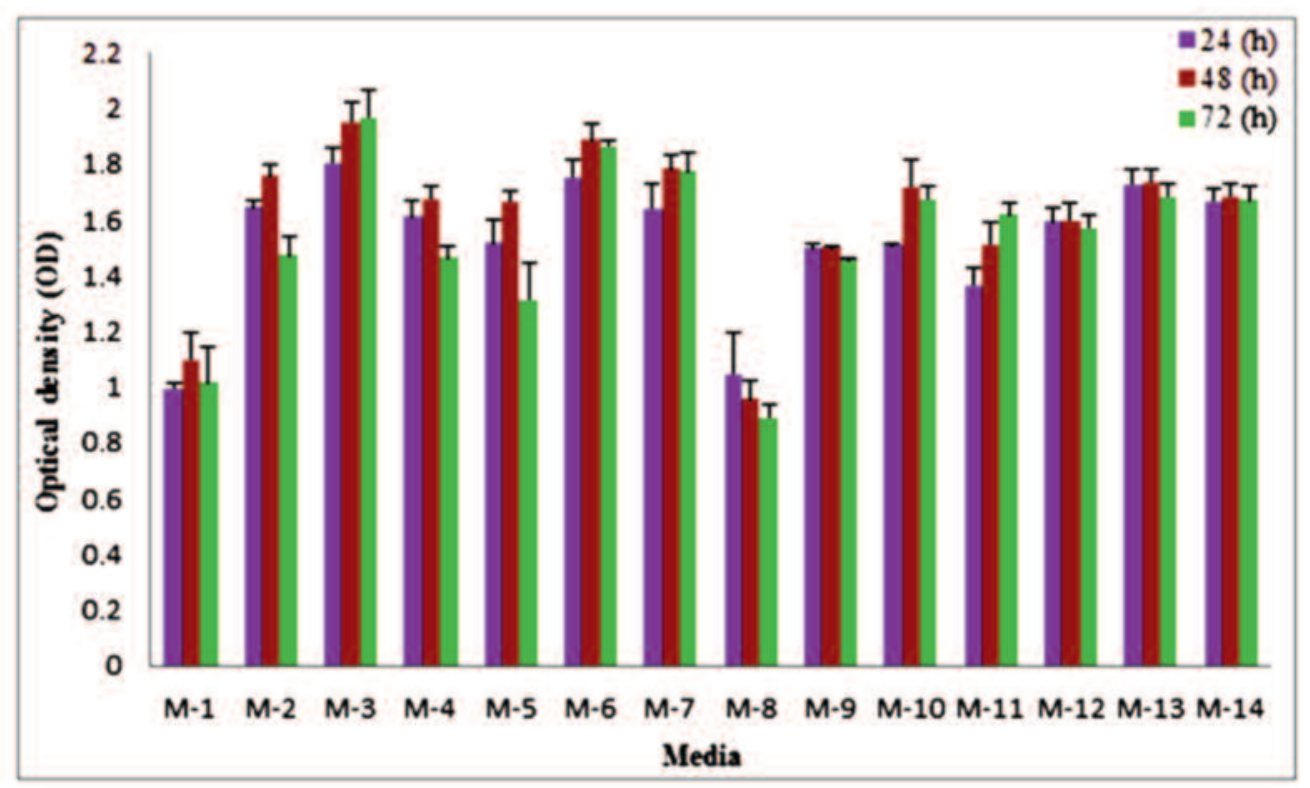

Fig. 1. Growth rates of $W$. hellenica DC06 in fourteen different media compositions with the mean \pm standard error.

taining $5 \mathrm{~g} / 1$ and $10 \mathrm{~g} / 1$ radish was poor due to insufficient nutrients. For sugar optimization, glucose (10 g/l and $20 \mathrm{~g} / \mathrm{l})$ and sucrose $(20 \mathrm{~g} / \mathrm{l})$ were tested with $20 \mathrm{~g} / \mathrm{l}$ radish and $10 \mathrm{~g} / \mathrm{l}$ yeast extract. Glucose $(20 \mathrm{~g} / \mathrm{l})$ supplementation yielded a higher growth rate than sucrose. With respect to yeast extract, we tested $5 \mathrm{~g} /$ land $10 \mathrm{~g} /$ lyeast extract with $20 \mathrm{~g} / 1$ radish and $20 \mathrm{~g} / 1$ glucose. Between these two compositions, media containing $10 \mathrm{~g} / \mathrm{l}$ yeast extract resulted in better growth compared with $5 \mathrm{~g} / \mathrm{l}$ yeast extract (Table I).
Fig. 2 shows a comparison of the growth of the analyzed strain in MRS and optimal media. The optical density of $W$. hellenica DC06 cultivated in optimal medium reached 1.8 $\left(1.066 \times 10^{10} \mathrm{CFU} / \mathrm{ml}\right)$ after incubation for $24 \mathrm{~h}$, which was slightly less compared to bacterial growth in MRS medium (optical density, 2.2 and $1.44 \mathrm{x} 1010 \mathrm{CFU} / \mathrm{ml}$ ) under the same conditions. Further, in addition to being edible, the new medium was approximately four times cheaper compared to MRS medium.

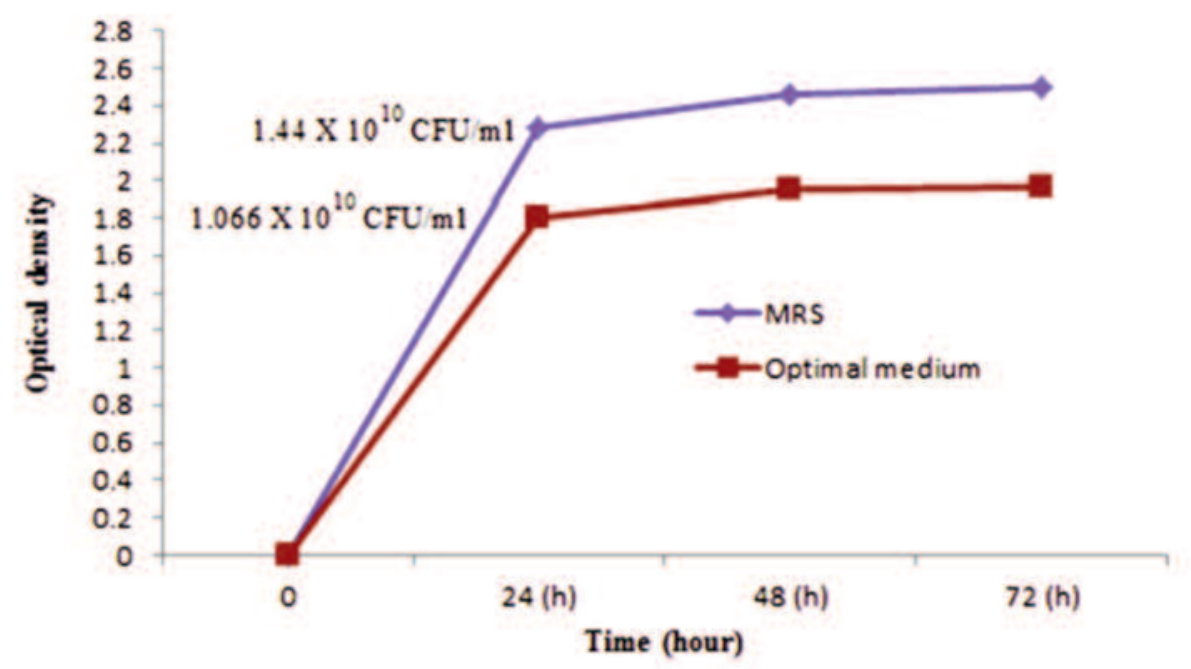

Fig. 2. Growth of $W$. hellenica DC06 in optimal medium (medium-3) compared with MRS medium. 
Fermentation of major ginsenoside $\mathrm{Rb} 1$ by $W$. hellenica DC06 in newly developed medium $W$. hellenica DC06 showed strong activity in newly developed medium in terms of ginsenoside biotransformation. Ginsenoside Rb1 was converted into pharmacologically active ginsenoside $\operatorname{Rg} 3$ by hydrolysis of two glucose units at the C-20 position of the ginsenosideaglycone. As shown in Fig. 3A, most of the ginsenoside Rb1 was transformed by $W$. hellenica DC06 into Rg3 within 5 days of fermentation and after 7 days of fermentation almost all ginsenoside Rb1 was transformed and converted into Rg3 (Fig. 3A). Ginsenoside Rb1 transformation ability of $W$. hellenica DC06 in MRS medium was compared with that of developed medium.As shown in Fig.3B, transformation activity of $W$. hellenica DC06 was weak, when $W$. hellenica DC06 was cultured in MRS medium with ginsenoside Rb1. After 7 days of fermentation in MRS medium $W$. hellenica transformed most of the ginsenoside $\mathrm{Rb} 1$ into Rd by hydrolysis of one glucose units at the C-20 position of the ginsenosideaglycone, but they could not transformed ginsenoside Rb1 to Rg3 in MRS medium. The effect of radish medium for biotransformation of ginsenoside Rb1 was also investigated but there was no effect of radish medium without lactic acid bacteria for Rb1 transformation (Fig. 3C).

The biotransformation of major ginsenoside Rb1 by $W$. hellenica DC06 was confirmed using quantitative HPLC analysis (Fig. 4). The peaks with retention times of 17.47, 21.35, $27.03,28.6,30.8$, and $31.16 \mathrm{~min}$ correspond to ginsenosides
Rb1, Rd, F2, Rg3, CK, and Rh2, respectively (Fig. 4A). Fig. $4 \mathrm{~B}$ shows the control of ginsenoside Rb1. The peak for ginsenosides Rb1 was no longer present within 7 days fermentation and one new peak that had retention time consistent with those of $\operatorname{Rg} 3$ was observed (Fig. 4C). Figure 4C showed the metabolite of ginsenoside Rb1 by $W$. hellenica DC06 that was grown in newly developed medium. W. hellenica DC06 produced ginsenoside $\mathrm{Rg} 3$ from $\mathrm{Rb} 1$ by hydrolysis of two glucose units at the $\mathrm{C}-20$ position of the ginsenosideaglycone. The transformation of ginsenosideRb1 by $W$. hellenica DC06 in MRS medium was also confirmed using quantitative HPLC analysis (Fig. 4D). After 7 days of fermentation, ginsenoside Rb1 was decomposed and converted into Rd by $W$. hellenica but they did not produce pharmacologically active minor ginsenoside Rg3 when used MRS medium (Fig. 4D). So, from TLC and HPLC data it is confirmed that $W$. hellenica DC06 showed strong activity in newly developed medium regarding on major ginsenoside $\mathrm{Rb} 1$ biotransformation into pharmacologically active minor ginsenoside Rg3.

The growth results showed that growth of $W$. hellenica DC06 with developed media containing $20 \mathrm{~g} / 1$ radish, $20 \mathrm{~g} / 1 \mathrm{glu}-$ cose, and $10 \mathrm{~g} / \mathrm{l}$ yeast extract supported sufficient growth that was slightly less than that obtained with the much expensive and non-edible MRS medium. The new medium containing only $10 \mathrm{~g} / \mathrm{l}$ yeast extract as a protein source compared with MRS medium which contains $25 \mathrm{~g} / 1$ of complex nitrogen sources. Yeast extract as a protein sourcewas usedin this study because of its relatively cheap cost compared with pro-

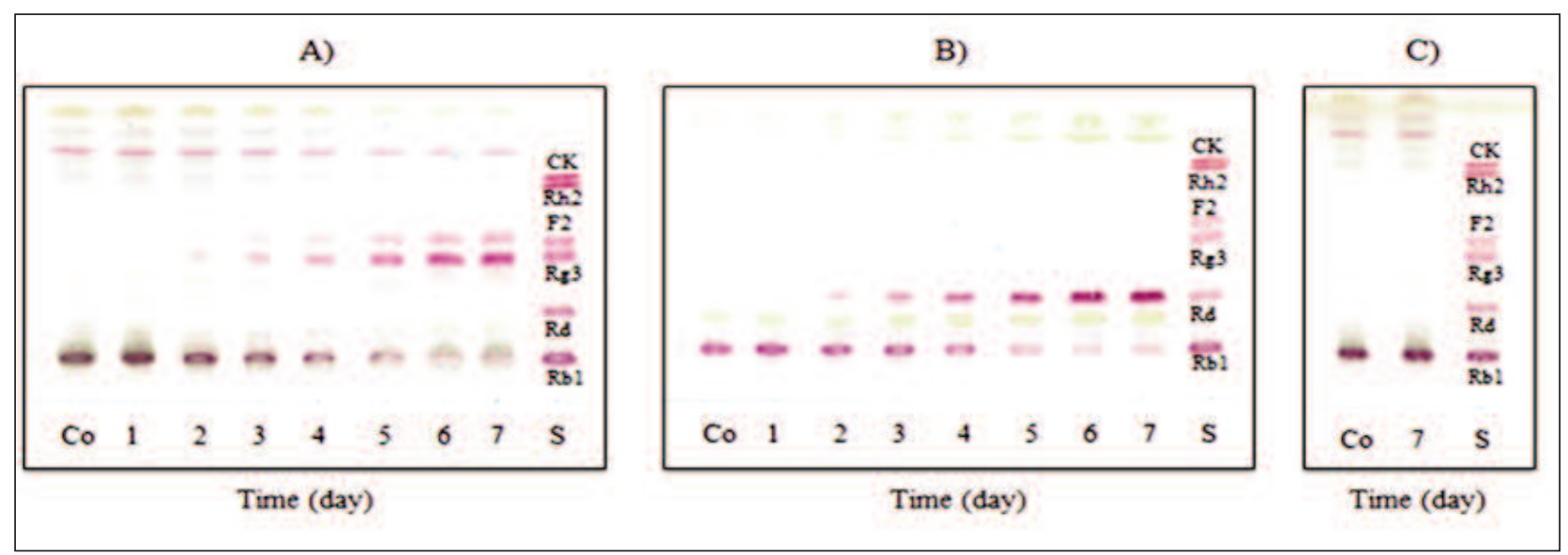

Fig. 3. TLC profile of transformation of major ginsenoside Rb1 by $W$. hellenica DC06.A), fermentation of ginsenoside Rb1 in new medium; B), fermentation of ginsenoside Rb1 in MRS medium; C), fermentation of ginsenoside Rb1 in new medium without $W$. hellenica $\mathrm{DC06}$ (Co = control; and S =saponin standards). 


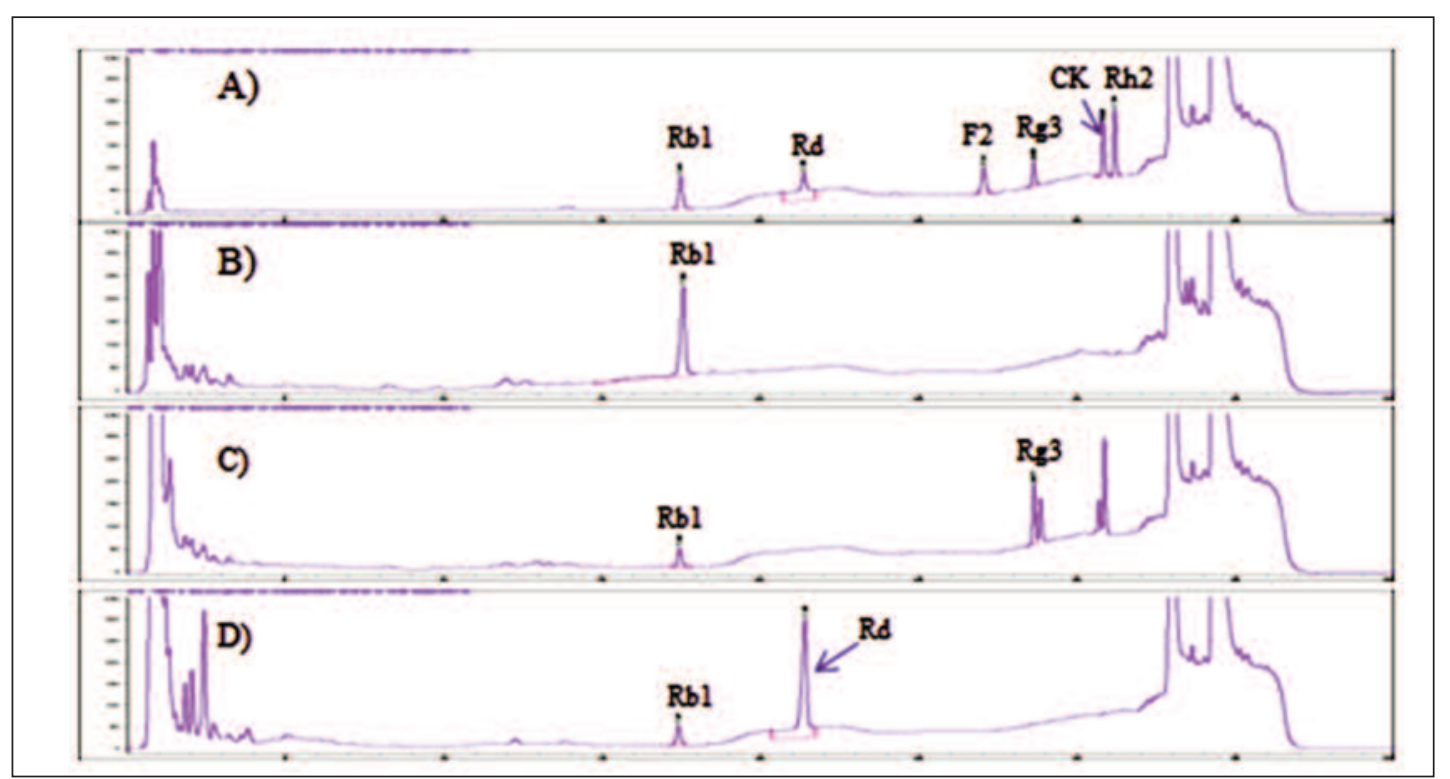

Fig. 4. HPLC profile of transformation of major ginsenoside Rb1 by $W$. hellenica DC06 in newly developed medium.A) Ginsenoside standards; B) ginsenoside Rb1 control; C) fermentation of ginsenoside Rb1 by $W$. hellenica in new medium and D) fermentation of ginsenoside Rb1 by $W$. hellenica in MRS medium.

tein sources used for MRS media, namely, peptospecial and beef extract. In addition, yeast extract is known to be a rich source of vitamins. Radishes also contain sufficiently high levels of protein at $680 \mathrm{mg}$ per $100 \mathrm{~g}$ of fresh root (USDA National Nutrient data base). Thus, radish also served as a protein source in the optimized medium.

While MRS medium contains several different types of supplemented micronutrients, no such micronutrientswere directly added to the new medium, and it only contained radish with carbon and protein sources. Thus, it was assumed that radish supplied necessary micronutrients because it contains sufficient amounts of sodium, potassium, calcium, magnesium, phosphorus, iron, zinc, and different vitamins (USDA National Nutrient data base), all of which may have contributed to its suitability for growing LAB.

Radishes contain significant amount of carbohydrates; however, glucose was used as a supplemental carbon source in the optimized medium because it produced better growth compared with sucrose. We also tested cabbage during development of the optimized medium (Table I); however, it facilitated less growth compared with radish.

Most LAB strains have similar growth requirements. So, new medium can be a good candidate for cultivation of other LABs. Moreover, the new medium was less expensive com- pared to MRS media: 1 liter of the new medium cost approximately 3 USD while 1 liter of MRS broth purchased from Sigma-Aldrich costs more than 10 USD. Another less using medium for cultivation of Lactobacillus is Tomato Juice Broth but it is around 10 times more expensive than new medium and 3 times more expensive than MRS medium according to price of DIFCO.Another important feature of the new medium is that it can be consumed by humans, and thus may be used by the food industry for cultivation of LAB strains. Along these same lines, there have been several efforts reported to date to develop an economical medium for LAB growth, including Lactobacillus fermentum (Gao et al., 2009), Lactobacillus rhamnosus (Berecka et al., 2010) and Lactobacillus plantarum (Horn et al., 2005). However, all of these media types are also more expensive than the newly described optimized medium.

Ginsenoside, a major component of ginseng, has been reported to exhibit various biological effects (Cho et al., 2006; Huq et al., 2015a). Minor ginsenosides are more active than intact major ginsenosides(Huq et al., 2015a). Therefore, many researchers have attempted to convert major ginsenosides to more active minor ginsenosides (Huq et al., 2014; Huq et al., 2015a,b). Minor ginsenoside Rg3 has strong anti-metastatic (Mochizuki et al., 1995), tumor-suppressing (Shinkai et al., 1996), hepatoprotective (Lee et al., 2005), neuroprotective (Tian et al., 2005) and vasodilating 
effects (Kim et al., 2003). This pharmacologically active ginsenoside $\operatorname{Rg} 3$ is not available in ginseng plant. In this study newly developed medium was used to produce available ginsenoside Rg3 from major ginsenoside Rb1 by $W$. hellenica DC06. Fermentation of ginsenoside Rb1 by $W$. hellenica $\mathrm{DC} 06$ using newly developed media produced Rg3 by hydrolysis of two glucose units at the C-20 position of the ginsenoside $\mathrm{Rb} 1$ aglycone, while used MRS medium for fermentation of ginsenoside Rb1 by $W$. hellenica DC06, the activity of biotransformation was decreased and W. helleni$c a$ DC06 produced Rd by hydrolysis of one glucose unit at the $\mathrm{C}-20$ position of the ginsenosideaglycone. The new medium may be enhanced the production of beta-glucosidase enzyme by $W$. hellenica DC06 that can easily convert the major ginsenoside Rb1 into pharmacologically active minor ginsenoside Rg3.

In conclusion, the results of this study demonstrated that media containing radishes may provide a good alternative to MRS for growing $W$. hellenica DC06 and other LAB strains with similar nutritional requirements. In addition, the new optimized medium may be potentially useful for industrial cultivation of $W$. hellenica DC06 and other lactic acid bacteria, especially in the food industry and for the synthesis of pharmacological active ginsenoside Rg3.

\section{Acknowledgements}

This research was supported by the Basic Science Research Program through the National Research Foundation of Korea (NRF) (\#20110015122), and is also supported by a grant from the Korea Institute of Planning and Evaluations for the Technology of Food, Agriculture, Forestry, and Fisheries (\#111035-03-1-SB010).

\section{References}

Berecka MP, Wasko A, Wiater MK, Podlesny M, Targonski Z and Komar AK (2010), Optimization of medium composition for enhancing growth of Lactobacillus rhamnosus PEN using response surface methodology, Polish journal of Microbiol. 59:113-118.

Cai Y, Benno Y, Nakase T and Oh TK (1998), Specific probiotic characterization of Weissellahellenica DS-12 isolated from flounder intestine, J. Gen. Appl. Microbiol. 44: 311-316.
Cho W, Chung W, Lee S, Leung A, Cheng C and Yue K (2006), Ginsenoside Re of Panax ginseng possesses significant antioxidant andanti-hyperlipidemic efficacies in streptozotocin-induced diabeticrats, Eur. J. Pharmacol. 550: 173-179.

De Man JC, Rogosa M and Sharpe ME (1960), A medium for the cultivation of lactobacilli, J. Appl. Bacteriol. 23: $130-135$.

Gao X, Qiao SY and Lu WQ (2009), Determination of an economical medium for growth of Lactobacillus fermentum using response surface methodology, Lett. Appl. Microbiol. 49: 556-561.

Horn SJ, Aspmo SI and Eijsink VGH (2005), Growth of Lactobacillus plantarum in media containing hydrolysates of fish viscera, J. Appl. Microbiol. 99: 1082-1089.

Huq MA, Kim YJ, Min JW, Bae KS and Yang DC (2014), Use of Lactobacillus rossiae DC05 for bioconversion of the major ginsenosides Rb1 and Re into the pharmacologically active ginsenosides $\mathrm{CK}$ and Rg2, Food Sci. Biotechnol. 23: 1561-1567.

Huq MA, Siraj FM, Kim YJ and Yang DC (2015a), Enzymatic transformation of ginseng leaf saponin by recombinant $\beta$-glucosidase (bgp1) and its efficacy in an adipocyte cell line, Biotechnology and Applied Biochemistry DOI: 10.1002/bab.1400.

Huq MA, Kim YJ, Hoang VA, Siddiqi MZ and Yang DC(2015b), Paenibacillusginsengiterrae sp. nov., a ginsenoside-hydrolyzing bacteria isolated from soil of ginseng field, Archives of Microbiology 197: 389-396.

Huq MA, Kim YJ, Min JW, Siraj FM, Siddiqi MZ and Yang DC (2015c), Enzymatic transformation of the major ginsenoside $\mathrm{Rb} 1$ to compound $\mathrm{K}$ by Weissella hellenica DC06, Indian Journal of Biotechnology 14: 270275.

Kim MJ, Seo HN, Hwang TS, Lee SH and Park DH (2008), Characterization of exopolysaccharide (EPS) produced by Weissella hellenica SKkimchi3 isolated from kimchi, J. Microbiology 46: 535-541.

Kim ND, Kim EM, Kang KW, Cho MK, Choi SY and Kim SG (2003), Ginsenoside Rg3 inhibits phenylephrine- 
induced vascular contraction through induction of nitric oxide synthase, Br. J. Pharmacol. 140: 661-670.

Lee HU, Bae EA, Han MJ and Kim DH (2005), Hepatoprotective effect of 20(S)-ginsenosides Rg3 and its metabolite 20(S)-ginsenoside Rh2 on tert-butyl hydroperoxide-induced liver injury, Biol. Pharm. Bull. 28: 1992-1994.

Liew SL, Ariff AB, Raha AR and Ho YW (2005), Optimization of medium composition for the production of a probiotic microorganism, Lactobacillus rhamnosus, using response surface methodology, Int. J. Food Microbiol. 102: 137-142.

Masuda Y, Zendo T, Sawa N, Perez RH, Nakayama J and Sonomoto K (2012), Characterization and identification of weissellicin $\mathrm{Y}$ and weissellicin $\mathrm{M}$, novel bacteriocins produced by Weissella hellenica QU 13. J. Appl. Microbiol. 112: 99-108.

Mochizuki M, Yoo YC and Matsuzawa K (1995), Inhibitory effect of tumor metastasis in mice by saponins, ginsenoside Rb2, 20(R)- and 20(S)-ginsenoside Rg3, of red ginseng, Biol. Pharm. Bull. 18: 1197-1202.

Rogosa M, Franklin JG and Perry KD (1961), Correlation of vitamin requirements with cultural and biochemical characters of Lactobacillus sub, Appl. Microbiol. Biotechnol. 24: 473-482.
Shinkai K, Akedo H, Mukai M, Imamura F, Isoai A, Kobayashi M and Kitagawa I (1996), Inhibition of in vitro tumor cell invasion by ginsenoside Rg3. Japan J. Cancer Res. 87: 357-362.

Tian JW, Fu FH, Geng MY, Jiang YT, Yang JX, Jiang WL, Wang CY and Liu K (2005), Neuroprotective effect of 20(S)-ginsenoside $\mathrm{Rg} 3$ on cerebral ischemia in rats, Neuroscience Letters 374: 92-97.

USDA National Nutrient data base, Radish (Raphanussativus), Fresh, raw, Nutrition Value per $100 \mathrm{~g}$.

Wells GAH, Scott AC, Johnson CT, Gunning RF, Hancock RD, Jeffrey M, Dawson M and Bradley R (1987), A novel progressive spongiform encephalopathy in cattle, Vet. Re. 121: 419-420.

Zhang J and Greasham R (1999), Chemically defined media for commercial fermentations, Appl. Microbiol. Biotechnol. 51: 407-421.

Received: 26 April 2016; Revised:06 June 2016; Accepted: 28 July 2016. 